УДК : 81’243+37.016:821:81’42

DOI: $10.24144 / 2617-3921.2020 .18 .321-330$

Olena Kuschnirtschuk старший викладач кафедри німецької філологї ДВНЗ «Ужгородський національний університет» orcid.org/0000-0002-1968-513X м. Ужсгород, Україна, +38 (050) 6763774 lena240572@gmail.com

\title{
Lernen an Hand der literarischen Texte
}

Анотація. Метою наукової статі $е$ висвітлення важливості використання тексту та роботи з текстом як иентральної складової навчального контексту іноземної мови, зокрема, важливості роботи $з$ текстом для формування мовних компетенцій. Літературний текст розглядається як методично-дидактичний засіб вивчення іноземної мови. Важливість та необхідність використання літературних текстів у прочесі навчання іноземній мові пояснюється декількома факторами. Літературний текст виступає, по-перше, джерелом лінгвокультурологічної інформачії про народ, мова якого вивчається (історія, культура, соціальний устрій, економічні та політичні характеристики тощо), та, по-друге, джерелом знань про функиіонування мовної системи та ї̈ прояви у період створення літературного твору. Необхідність використання літературного тексту на заняттях з іноземної мови пояснюється також тим, що робота з текстом сприяє формуванню світогляду, власної думки, допомагає розвивати висловлювання щодо різноманітних ситуачій іноземною мовою. Робота 3 текстом формує, розвиває та вдосконалює всі мовні компетениії, якщзо підбір тексту здійснено методично правильно. Основними критеріями відбору тексту для заняття з іноземної мови $е$ відповідність рівню знань та відповідність навчальній меті на конкретному етапі. Кожен літературний текст фактично є невичерпним джерелом інформачії, $i$ саме викладач повинен навчити студента, як июю інформацію потрібно виокремлювати, аналізувати та вміти використовувати для ведення дискусії. Робота над будь-яким літературним твором повинна бути ретельно продуманою та логічно спланованою. На початковому етапі вивчення іноземної мови одним з найкращих літературних жанрів виступає казка, в якій присутні образність, абстракиія та повчання. Казка, зміст якої несе інформацію про національномовну картину світу, викликає особливий інтерес до ї̈ опрацювання. На основі обраної казки, як і будь-якого іншого літературного тексту, пропонуються ряд практичних завдань, які рекомендується виконати на заняттях з іноземної мови перед, під час та, відповідно, після ознайомлення із змістом тексту. Такий вид діяльності дозволяє розкривати творчий потенціал 
студентів, спонукаючи їх до креативного мислення та осмислення прихованого змісту. Тому в навчальному процесі такому аспекту як читання, через яке ми пізнаємо як мову, так і культуру іншого народу, слід приділяти підвищену увагу.

Ключові слова: заняття з іноземної мови, робота з текстом, мовні компетенияї.

Abstract. The purpose of this scientific article is to highlight the importance of using the text and working with it as a central component of the educational context of a foreign language, and particularly the importance of working with the text for the formation of language competencies. Literary text is considered as a methodological and didactic tool for learning a foreign language. The importance and necessity of using literary texts in the process of learning a foreign language is explained by a few factors. Literary text is, firstly, a source of linguistic and cultural information about the people whose language is being studied (history, culture, social order, economic and political characteristics, and secondly, a source of knowledge about functioning of the language system and its manifestations during the creation of a literary work. The necessity to use a literary text in foreign language classes is also explained by the fact that working with the text contributes to the formation of worldview, own opinion, helps to develop statements about various situations in a foreign language. Working with the text forms, develops and improves all language competencies, if the text is selected methodically correctly. The main criteria for selecting a text for a foreign language lesson are compliance with the level of knowledge and compliance with the learning objective at a particular stage. Each literary text is in fact an inexhaustible source of information, and it is the teacher whose main responsibility is to teach a student how to extract, analyze and be able to use this information for discussion. Work on any literary text must be thoroughly thought through and logically planned. At the initial stage of learning a foreign language, one of the best literary genres is a fairy tale, which involves imagery, abstraction and teaching. It is particularly interesting to elaborate on a fairy tale, the content of which carries information about the national-linguistic picture of the world. Based on the selected fairy tale, just as on any other literary text, a number of practical tasks are offered, which are recommended to be performed in foreign language classes before, during and after getting acquainted with the content of the text. This type of activity allows you to reveal the creative potential of students, encouraging them to think creatively and comprehend the hidden content. Therefore, the reading aspect, through which we learn both the language and culture of another people, should be given special attention in the educational process.

Keywords: foreign language classes, work with text, language competencies.

Einleitung. Der Textarbeit wird in der modernen Didaktik eine entscheidende Rolle zugewiesen. Im Fremdsprachenunterricht mobilisiert die Textarbeit Wissen und 
Können, das teilweise spezifisch für den Fremdsprachenunterricht, teilweise sprachenübergreifend ist. Die Aktualität des Forschungsthemas wird dadurch erklärt, dass Lesen ein komplexer Vorgang ist, der aus mehreren kontextabhängigen Teilprozessen auf der Wort-, Satz- und Textebene besteht. Deshalb gehört der Textarbeit eine wichtige Rolle beim Erwerb der Fremdsprache und dieses Thema gehört zum Mittelpunkt der wissenschaftlichen Untersuchungen in Bezug auf die Effektivität der Aufgaben und Übungsarten, die an Hand der Textarbeit erfüllt werden.

Der wichtige Grund dafür, dass der Literatur im Fremdsprachenunterricht der hohe Stellenwert gehört, ist, dass die literarischen Texte 1) als Informationsquelle über Geschichte, Kultur, Lebensweise und Weltvorstellungen fremder Völker, deren Sprache erlernt wird und 2) als eine der Grundlagen für die Förderung interkultureller Beziehungen, für den Austausch humanistischer Kulturgüter und somit für die Völkerverständigung ist [4, S. 11]. Der nächste sehr wichtige Grund für den Texteinsatz in den Fremdsprachenunterricht ist, dass man dabei mit zwei Medien der nationalen Kultur hat: 1) mit der Literatur als einer besonderen Kunstart und 2) mit der Sprache, als mit deren „Baustoff“. Ohne sich in die Literatur der erlernenden Sprache einzuleben, kann man die lebendige Substanz einer fremden Sprache kaum erfassen [4, S. 16]. Der Einsatz der Textarbeit im Deutschunterricht bei der Vorbereitung der Germanistik-Studenten ist ein erfolgreiches Verfahren der Aneignung des Gesamtkomplexes der Sprachkenntnisse, deshalb wird der Textarbeit eine wichtige Bedeutung zugeschrieben.

Der literarische Text dient seit den 1970er Jahren im Fremdsprachenunterricht als Diskussionsgrundlage für eine sprachliche Beschäftigung mit den Problemen der Zielsprache. Vor diesem Hintergrund wurden literarische Texte ausgewählt, die „Perspektiven des modernen Lebens aufzeigen und Kunde von den Erscheinungen und Hintergründen der Kultur und den wirtschaftlichen, technischen und sozialen Befindlichkeiten geben“ [10, S. 1217]. Der zu dieser Zeit dominierende kommunikative Ansatz mit seinem übergeordneten Lernziel der „kommunikativen Kompetenz" stand ganz unter dem Zeichen des Pragmatismus, weshalb Literatur im Fremdsprachenunterricht nicht in ihrem ästhetischen Wert gewürdigt, sondern überwiegend zu Sprachlernzwecken herangezogen wurde [10, S. 1218].

In den 1980er Jahren bestand schließlich wieder Einigkeit darüber, Literatur im Fremdsprachenunterricht einzusetzen [19, S. 22]. Mit den Forschungszweigen der interkulturellen Germanistik und der damit einhergehenden Hermeneutik der Fremde erlangen literarische Texte unter dem Aspekt der Bedingungen fremdkultureller Rezeption einen neuen Stellenwert [4, S. 7].

Es gilt als besonders gewinnbringend, den Schülern fiktionale Texte nahezubringen, da diese in ihrem Bezug zur Welt „offener“ sind und subjektiv gedeutet werden könnten. Es findet beim Rezeptionsprozess eine Vermischung von eigenem Weltverständnis und der fremdkulturellen Welt des Textes statt. Dies ermöglicht dem Lerner einen direkteren emotionalen Bezug zum Gelesenen [5, S. 81]. 
Das Forschungsobjekt ist Einsatz der literarischen Texte als Arbeitsmethode im Fremdsprachenunterricht.

Zum Forschungsgegenstand wurden die Rolle der Textarbeit im Deutschunterricht, Beschreibung der praktischen Anwendung der literarischen Texte im Deutschunterricht und ihre Funktionen beim Fremdsprachenerwerb.

Methodisches Verfahren. Der Beitrag gründet auf den aktuellen Veröffentlichungen der Wissenschaftler zum Forschungsthema und auf der Erfahrung des Einsatzes von der Textarbeit im Deutschunterricht an der Fakultät für Fremdsprachen der Nationalen Universität Uschhorod.

Resultate und Diskussionen. Diese werden nach bestimmten Kriterien für den Unterricht ausgewählt oder erstellt. Wie diese Texte im Unterricht verwendet werden und welche Lehr- und Lernziele mit der Textarbeit verbunden sind, gehört in den Bereich der Methodik und Didaktik [2, S. 406]. Die „Arbeit an, mit und nach Texten", wie Hans-Eberhard Piepho genannt hat, gehört zum Bereich der sogenannten methodisch-didaktischer Kompetenz [12, S. 5-6].

Der Begriff „Textarbeit“ ist eine Besonderheit der deutschsprachigen Fremdsprachendidaktik. Die Textarbeit wird häufig der interkulturellen Philologie zugerechnet. „Textarbeit ist jede intensive mündliche oder schriftliche Beschäftigung mit Texten, ihren Inhalten und dem darin enthaltenen Sprachmaterial“ [2, S. 403]. Der Begriff „Textarbeit“ definiert in der Regel nicht, sondern wird einfach gebraucht, wenn von der Arbeit mit Texten im Fremdspracheunterricht die Rede ist und bezeichnet im weitesten Sinne die sprachdidaktisch begründete Arbeit mit Texten und ist wohl deshalb so nützlich, weil er eine geradezu selbstverständliche Komponente der Spracharbeit im Unterricht beschreibt [3, S. 15].

Texte sind ein wesentlicher Bestandteil des Fremdsprachenunterrichts. Der Gebrauch der Schrift und vielfältige Formen der Textarbeit sind im Kontext des Erwerbs und des Erlernens fremder Sprachen seit der Antike gut dokumentiert. Die Lernenden orientierten sich an den Texten der als Vorbilder geltenden Autoren. Methoden, die im 19. und im 20. Jahrhundert die praktische Beherrschung der Sprache betonen, stellen Dialoge und alltagsbezogene Texte in den Mittelpunkt. Der kommunikative Ansatz bereicherte die didaktische Diskussion um die Forderung nach dem Einsatz authentischer Texte [10, S. 114-117].

Der literarische Text ist ein äußerst wichtiges Mittel der Modellierung des sprachlichen und landeskundlichen Usus der jeweiligen Fremdsprache, der eine konkrete Etappe der gesellschaftlich-politischen, kulturell-historischen und sozialökonomischen Entwicklung des betreffenden Landes widerspiegelt. Die Anwendung der literarischen Texte als ein bestimmtes sprachlich-landeskundliches Modell sichert die Authentizität der Fremdsprachennutzung, denn dieses Modell wird von einem begabten und kompetenten Muttersprachler (Autor des Textes) unter Verwendung eines authentischen Sprachstoffes geschaffen [8, S. 338]. Als authentische Texte werden in der Literatur solche Texte bezeichnet, die 
zielsprachlichen Kommunikationskontexten entstammen und sehr unterschiedlichen Textsorten angehören können: Lieder, Gedichte, Rezepte, Einkaufslisten u. a.

Bei der Vorbereitung der Textarbeit im Fremdsprachenunterricht scheinen solche didaktischen Fragen wichtig zu sein: die erste betrifft die Kriterien, nach denen die Texte ausgewählt werden, die andere betrifft die Merkmale dieser Texte, die sich durch die Textarbeit im Unterricht verändern (können).

Ein sehr wichtiger Grundsatz der didaktischen Interpretation literarischer Texte im Fremdspracheunterricht ist das Prinzip der zielgerichteten Textauswahl. Die zu interpretierenden Texte müssen vorbildlich in ihrer sprachlichen Gestaltung sein. Die Forderung nach der mustergültigen sprachlichen Gestaltung schließt ein, dass die deutsche Sprache in diesen Texten in all ihren Existenzformen und Ausdruckspotenzen nach Möglichkeit vertreten wird. Durch das wirkungsorientierte, tätigkeitsbezogene Behandeln der sprachlich-stilistischen Textgestaltung werden die Lernenden einerseits: zur höheren landeskundlichkommunikativen Kompetenz und andererseits zum produktiveren Sprachgebrauch erzogen [7, S. 141].

Das Problem der Kriterien für die Textauswahl wird in der didaktischen Literatur ausführlich diskutiert. Der Erwerb der Schriftsprache ist eine notwendige, jedoch keine hinreichende Bedingung für die Entwicklung von Lesekompetenz. Damit aus Lernenden, die gut lesen können, Lernende werden, die kompetent Texte bearbeiten und aus ihnen effektiv selbstgesteuert lernen, müssen weitere Bedingungen gegeben sein:

1. Für die effektive Textverarbeitung ist zwar von großer Bedeutung die Kapazität des Arbeitsgedächtnisses. Andere Merkmale und charakteristischen Aktivitäten guter Leser sind hingegen prinzipiell erlernbar.

2. Von den einzelnen Determinanten der Lesekompetenz wie Arbeitsgedächtnis, inhaltlichem Vorwissen, lexikalischem Zugriff, Wortschatz, (Lese-)Motivation, Kenntnis von Textmerkmalen, Lesestrategien und Selbstregulation beim Lesen sind es vor allem Motivationsmerkmale und die Prozessmerkmale der Selbstregulation, auch die Kenntnis von Textmerkmalen.

3. Der Lehrer fragt sich, ob der Text Anknüpfungsmöglichkeiten in die Lebenserfahrung der Schüler und Anregungen zum Weiterlesen bietet, welche sprachlichen Schwierigkeiten im Text enthalten sind und ob die Textsorte den Schülern geläufig ist.

4. Bestimmung der Lernsituation: es ist $\mathrm{zu}$ bestimmen, welchen Vorbereitungs- und Durchführungsaufwand der Text erfordert, welche Möglichkeiten er für systematische Spracharbeit und zur Entwicklung freierer Übungen zum Sprechen / Schreiben bietet [11, S. 1217].

Bei der Unterrichtsvorbereitung und bei der Textauswahl soll vom Lehrer geklärt werden:

- ob der Text authentisch ist und ob er ein Problem berührt,

- was die Schüler interessiert,

- ob die Schüler das Wesentliche des Textes erschließen können, 
- ob die Problematik aus anderen Fächern und anderssprachigen Texten bekannt ist,

- ob der Text Anlass zum Handeln bietet,

- ob er einen Bezug zu den Themen im Lehrwerk hat,

- ob der Text im weitesten Sinn eine pädagogische/emanzipatorische Funktion spielt,

- ob der Text wichtige, wissenswerte Informationen enthält,

- ob er die Emotionen der Schüler anspricht,

- ob der Text eine Auswirkung auf das Leben der Schüler hat [9, S. 152].

Falls der Lehrer die meisten Fragen mit „Ja“ beantwortet, kann er sicher sein, dass der angegebene Text alle vier oben genannten Faktoren genug tut und dass er alle Anforderungen der modernen Textarbeit erfüllt.

Die Kriterien der Textauswahl werden in der Regel zielgruppenspezifisch vorgeschlagen. Als Beispiel für die Anfangsstufe gelten die Geschichten, die Kinder faszinieren und in der Fremdsprache angenommen werden. Die ausgewählten Texte sollen einigen der folgenden Kriterien entsprechen:

1) Thema und Sprache der Geschichte müssen kindgerecht sein;

2) die Geschichte muss einen Spannungsbogen haben (Anfang, Problem, Problemlösung, Ende);

3) die Geschichte muss der vortragenden Person gefallen;

4) die Geschichte sollte den Kindern eine Möglichkeit bieten, sich mit den Figuren zu identifizieren, mitzufuhlen, weiterzudenken;

5) bei den Geschichten sollte die Aussage der Bilder und des Textes ubereinstimmen. Bilder und Illustrationen müssen für die Kinder verstehbar sein und die Neugier wecken;

6) für die jungen Lernenden und Anfänger empfehlen sich Geschichten, deren sprachliche Muster sich wiederholen. Die Kinder werden diese Wiederholung recht bald als Chunks abspeichern und selbst wiedergeben können;

7) die Geschichte sollte lustig, spannend und Fantasie anregend sein [7, S. 132].

Also, es handelt sich um Kriterien, die sich ebenso auf die Texte wie auf deren Gebrauch beziehen und mit deutlicher Orientierung am Zielpublikum formuliert werden.

Diese Kriterien sollen den Lehrern bekannt sein, wenn sie Texte in Lehrwerken vorfinden und mit diesen arbeiten möchten. Wenn man Lehrwerke ergänzt, indem man selbst zusätzliches Material, zusätzliche Texte auswählt und ihre didaktische Brauchbarkeit beurteilt, benötigt man ebenfalls Kriterien zur Textbeurteilung.

Eine wichtige Frage ist dabei auch der Umgang mit den ausgewählten Texten im Unterricht. Die Lehrer verwenden Texte, indem sie Lehr- und Lernsituationen schaffen, in denen ihre Schüler anhand dieser Texte lernen. Dafür werden die Arbeitsanweisungen formuliert, Aktivitäten aus Lehrwerken werden ausgewählt oder werden diese adaptiert, zusätzliches Arbeitsmaterial wird erstellt und überlegt, 
welche Lehr- und Lernziele erreicht werden soll. Eine wichtige Voraussetzung für diese didaktische Arbeit ist, dass die Lehrkräfte die sprachlichen, funktionalen und situativen Charakteristika von Textsorten kennen [1, S. 146-147].

Wolfgang Hallet beschreibt fachdidaktische Kompetenz als die Fähigkeit, „fachliche Inhalte so auszuwählen und zuzuschneiden, dass sie aktive Lern- und Wissenserwerbprozesse anhand zentraler und exemplarischer fachlicher Inhalte ermöglichen" " 6 , S. 45].

Jedes konkrete literarische Werk ist ein eigentümliches Material zur Aneignung der in der realen Textwirklichkeit aktualisierten sprachlichen Einheiten. „Für viele große Dichter verschiedener Nationen war und ist die Sprache nicht nur „ein Werkzeug“, sie verkörpert die Seele und das Schicksal ihres Volkes“ [7, S. 133].

Jeder Text ist Mittelpunkt von verschiedenen künstlerischen und sprachlichen Substanzen, die außerhalb des Textes sowohl synchronisch als auch diachronisch funktionieren. Analytisch betrachtet und zum Zweck des Unterrichts lassen sich alle diese Substanzen einzeln interpretieren, d.h. künstlerische, sprachliche, synchronische und diachronische Kategorien können getrennt betrachtet werden [11, S. 1217].

Unter den sprachlichen Kategorien können auch extra- und intratextuale Elemente ausgegliedert werden: $\mathrm{zu}$ den ersten gehört das ganze dem Autor synchrone System der Muttersprache und die Sprache jener sozialen Gruppe, zu der der Autor gehört, zu den letzteren die im Text gebrauchte Rede mit allen ihren Formen und Arten [1, S. 157].

Eine besondere Rolle kommt der zweidimensionalen Intensität der Analyse beim Behandeln eines fremdsprachigen literarischen Textes zu. Der Lehrer muss im Prozess der didaktischen Textinterpretation bestrebt sein, die zwei Wirklichkeiten zu verflechten: die vom Autor erdachte Welt des künstlerischen Textes und die reale Welt der Textrezipienten in ihren nationalen, regionalen, zeitlichen $u$. a. Dimensionen [13, S. 35].

Der Texteinsatz und der Umgang mit den literarischen Texten im Fremdsprachenunterricht hat schon gut ausgearbeitete Methoden und Arbeitsformen, auch eine gute Erfahrung. An der Fakultät für Fremdsprachen der Nationalen Universität Uschhorod, bei der Ausbildung der Germanistik-Studenten wird die Textarbeit häufig eingesetzt. Die Arbeit am Text ist immer sorgfältig geplant und überlegt. Als Beispiel der aller möglichen Formen der Textarbeit im Deutschunterricht im 1. Studienjahr ist der Einsatz der Märchentexte. Die Erfahrung zeugt davon, dass am besten dazu die Märchen passen, die den ukrainischen Lernenden wenig bekannt sind. Am Anfang bekommen die Studenten eine Hausaufgabe - nach der Information über diese Märchen und deren Autoren im Internet suchen: was sie geschrieben haben, typische Merkmale ihrer Werke usw., damit sie auf die Diskussion mit den Autoren vorbereitet sind. Die Diskussion verläuft in der Form eines Interviews mit den Autoren. In der nächsten Etappe werden die Vermutungen über den literarischen Text gemacht. Das wird als 
Galgenspiel veranstaltet. Zuerst sollen die Lernenden erraten, welches Buch gelesen wird. Die Regeln sind die folgenden: für jeden Buchstaben eines Wortes wird ein Strich an die Tafel gezeichnet und man soll das Wort erraten. Das Erraten des Wortes erfordert von den Lernenden Kenntnisse über Buchstabenkombinationen, die auf Deutsch üblich sind. Die nächste Etappe beabsichtigt die Vermutungen über den Inhalt zu äußern. Das Ziel in dieser Phase ist, inhaltliche und sprachliche Aspekte des Märchens zu vereinfachen und den Studenten verständlich zu machen. Es werden verschiedene Sachen wie z. B. Titel des Märchens, Umschlag, graphisches Layout, Illustrationen, Klappentexte usw. benutzt. Es werden Vermutungen nach den Bildern gemacht: man zeigt ein paar Bilder aus dem Märchen und die Lernenden spekulieren über den Inhalt des Märchens.

Eine interessante Übungsart ist die Vermutungen nach Schlüsselwörtern zu machen. Bevor man das Märchen zu lesen beginnt, werden Schlüsselvokabel aus dem Text gewählt, diese auf Karten aufgeschrieben und die Studenten verfassen in Gruppen ihre eigene Geschichte. Es werden Hypothesen über den Inhalt aufgebaut, die erfüllt werden können oder nicht erfüllt werden müssen. Dann werten sie zusammen alle Geschichten aus und vergleichen sie.

Diese Übungsarten und Aufgaben entwickeln bei den Lernenden die Fähigkeit zu vermuten, die Handlungsfolge fortzusetzen, die Phantasie und die Vorstellungen zu bereichern. Diese Arbeitsformen haben sich als sehr positiv und lernfördernd erwiesen.

Während des Lesens verläuft also verschiedene Textarbeit, es handelt sich vor allem um Fragen zum Textverständnis und Übungen, die an den Text anknüpfen. Z.B., die Übung „Wie war das?“, wobei verschiedene Sätze angegeben werden, die den gelesenen Märchenabschnitt repräsentieren. Die Studenten sollen die Sätze richtig zuordnen. Einige Übungen verlangen die Vervollständigung der Sätze dem Textinhalt nach, es werden auch Spielpausen vorgeschlagen, wobei man den Inhalt inszeniert. Es werden die Übungen: „Zusammenfassung nach dem Lehrer“ gemacht, diese Aktivität ist zum Üben der Fertigkeit Hören bestimmt; die Übung „Dramatisierung“, wobei die Lernenden die vorgeschlagene, ausgedachte Fortsetzung vorspielen und dabei ihre Rollen wörtlich gestalten; Übung zum Erlernen der neuen Wörter (jeder bekommt ein Wort, das er im Wörterbuch aussuchen soll und damit einen Satz bilden). Die Lernenden sollen eine Situation ausdenken, in die das Wort vorkommt. Die anderen sollen die Bedeutung des Wortes erraten. Zum Schluss kommt die gemeinsame Kontrolle, z.B., die Übung „Richtige Reihenfolge setzen“ oder "Sachen, die sich auf die Hauptfigur beziehen“: die Studenten bekommen Sätze aus dem Märchen in der zufälligen Folge. Sie sollen diese in die richtige Reihenfolge setzen, wie sie nacheinander im Märchen stehen. Nach der Lektüre schreibt der Lehrer ukrainische Vokabeln aus diesem Teil an die Tafel und die Studenten sollen die deutschen Äquivalente im Text aussuchen. Diese Übung wird als ein Wettbewerb organisiert. Noch eine Übung zur Kontrolle des Gelesenen: „Wer hat das gesagt?“" Es werden verschiedene Aussprüche aus dem 
Text angeboten. Die Studenten sollen entscheiden, welche Figuren diese Worte ausgesprochen haben.

Zum Schluss der Arbeit soll der Lehrer die Lernenden zur weiteren literarischen Arbeit motivieren und positive Aspekte des Lesens hervorheben. Den Studenten wird auch ein Fragebogen gegeben, in dem bewertet wird, wie sie die Arbeit mit dem Text und den Text selbst finden.

Schlussfolgerungen. Die Erfahrung zeugt davon, dass die Textarbeit im Fremdsprachenunterricht ein vielschichtiges Phänomen ist. Es werden bestimmte Aufforderungen zur Textauswahl gestellt. Der Lehrer soll den Text sorgfältig je nach den Unterrichtszielen und der Fähigkeiten und Fertigkeiten der Lernenden wählen. Beim Texteinsatz im Fremdsprachenunterricht wird viel schöpferisch und kreativ, nicht gedruckt und problematisch, sondern gern und hemmungslos gearbeitet, was auch dem erfolgreichen Lernen beiträgt. Der Texteinsatz motiviert zum Lernen, steigert das Interesse an den Sprachkenntnissen und formiert die kommunikativen Fähigkeiten und Fertigkeiten bei den Lernenden. Lernen an Hand der Texte bietet verschiedene Aufgaben- und Übungstypen an, die der erfolgreichen Sprachbeherrschung beibringen.

\section{LITERATUR}

1. Adamzik K. Textsorten und ihre Beschreibung. Textlinguistik. Einführungen. Tübingen: Narr, 2008. S. 145-176.

2. Barkowski H., Krumm H.-J. Fachlexikon Deutsch als Fremd- und Zweitsprache. Tübingen: Francke, 2010. $780 \mathrm{~S}$.

3. Bimmel P. Lernerautonomie und Lernstrategien. München: Langenscheidt, 2000. $208 \mathrm{~S}$.

4. Edelhoff Chr. Authentizität im Fremdsprachenunterricht. Authentische Texte im Deutschunterricht. Einführung und Unterrichtsmodelle. München: Hueber, 1995. S. 7-30.

5. Götz M. Schriftspracherwerb als grundschuldidaktisches Problem. LeseSchreibschwache. Vorbeugen, Erkennen, Helfen. Lese-Schreibschwache. Vorbeugen, Erkennen, Helfen. Würzburg: edition bentheim, 2004. S.75-94.

6. Hallet W. Didaktische Kompetenzen. Lehr- und Lernprozesse erfolgreich gestalten. Stuttgart: Klett, 2006. 213 S.

7. Hufeisen B. Mehr Sprachen? - Plur Cur! Berichte aus Forschung und Praxis zu Gesamtsprachencurricula. Baltmannsweiler: Schneider Verlag Hohengehren, 2016. S. 130-141.

8. Hufeisen B. Förderung des DaF-Unterrichts durch Mehrsprachigkeitskonzepte. Förderung der deutschen Sprache weltweit Vorschläge, Ansätze und Konzepte. Berlin/Boston: de Gruyter, 2019. S. 337350.

9. Hufeisen B., Thonhauser I. Authentische, didaktisierte und didaktische Texte - Überlegungen zur Textarbeit aus drei verschiedenen Perspektiven. 
Auf dem Weg zu einer Textsortendidaktik. Linguistische Analysen und text(sorten)didaktische Bausteine nicht nur für den fremdsprachlichen Deutschunterricht. Hildesheim, Zürich, New York: Olms, 2018. S. 149-164.

10. Hüllen W. Kleine Geschichte des Fremdsprachenunterrichts. Berlin: Schmidt, 2005. $254 \mathrm{~S}$.

11. Krumm H.-J. Lehrwerke im Deutsch als Fremd- und Deutsch als Zweitsprache-Unterricht. Deutsch als Fremd- und Zweitsprache. Ein internationales Handbuch. Berlin, New York: Edition, 2010. S. 1215-1226.

12. Piepho H.-E. Leseimpuls und Textaufgabe. Textarbeit im Deutschunterricht. Fremdsprache Deutsch. Jg. 2. 2005. S. 4-9.

13. Riedner R. Authentizität in der Fremdsprachendidaktik - kritische Anmerkungen $\mathrm{zu}$ einem problematischen Konzept. Deutsch als Fremdsprache. 2018. Jg. 55. S. 34-43.

УДК 81'243-25

DOI: $10.24144 / 2617-3921.2020 .18 .330-338$

Вікторія Леган

викладач кафедри іноземних мов ДВНЗ «Ужггородський національний університет» ORCID 0000-0003-0222-3120 м.Ужсгород, Україна, 0508849380 viktoriya.lehan@uzhnu.edu.ua

Наталія Годованець кандидат філософських наук,доиент дочент кафедри іноземних мов ДВНЗ «Ужггородський національний університет» ORCID 0000-0003-3429-6973 м.Ужггород, Україна, 0958789075 nataliia.hodovanets@uzhnu.edu.ua

\section{The notion of communicative competence in the context of foreign language learning}

Анотація. Стаття присвячена висвітленню та аналізу поглядів зарубіжних та вітчизняних учених та основні положення компетентності як структурного компонента іншомовної комунікативної компетентності. Розкривається сутність комунікативних понять у контексті оволодіння іноземними мовами. Здійснюється аналіз та порівняння структури та 